\title{
Wnt/Wingless Signaling in Drosophila
}

\author{
Sharan Swarup and Esther M. Verheyen \\ Department of Molecular Biology and Biochemistry, Simon Fraser University, Burnaby, British Columbia \\ V5A1S6, Canada \\ Correspondence: everheye@sfu.ca
}

The Wingless $(\mathrm{Wg})$ pathway represents one of the best-characterized intercellular signaling networks. Studies performed in Drosophila over the last 30 years have contributed to our understanding of the role of Wg signaling in the regulation of tissue growth, polarity, and patterning. These studies have revealed mechanisms conserved in the vertebrate Wnt pathways and illustrate the elegance of using the Drosophila model to understand evolutionarily conserved modes of gene regulation. In this article, we describe the function of Wg signaling in patterning the Drosophila embryonic epidermis and wing imaginal disc. As well, we present an overview of the establishment of the $\mathrm{Wg}$ morphogen gradient and discuss the differential modes of Wg-regulated gene expression.

-volutionarily conserved cell signaling pathEways regulate the development of metazoans through their reiterative implementation, both spatially and temporally. Wnt signaling represents one such pathway that has multiple, essential roles during both embryogenesis and adult homeostasis to regulate cell proliferation, cell polarity, and the specification of cell fate (for review, see Wodarz and Nusse 1998). Wnt genes encode secreted glycoprotein ligands that can act both as short-range signaling molecules and long-range morphogens, depending on the developmental context. Members of the Wnt family are defined by sequence homology to Wnt-1 (Nusse and Varmus 1982; Nusse et al. 1984), the first identified Wnt protein, rather than by functional homology. As such, subsequent to the identification of Wnt-1, diverse Wnt-regulated processes have been identified that when aberrantly regulated result in myriad diseases, ranging from developmental disorders to cancers. Thus far, 19 vertebrate Wnt family members have been discovered, of which there are seven homologs in Drosophila (Table 1). Much of our understanding of the role of Wnt proteins during development has come as a result of genetic analyses of the Drosophila wnt-1 (Dwnt-1) or wingless (wg) gene.

As the name suggests, the $w g$ gene is required to pattern the Drosophila wings and other adult body structures. It was originally identified through a hypomorphic allele, $w g^{1}$, which harbors a deletion in a regulatory element of the gene and causes the variable transformation of the adult wing(s) to thoracic notum (Sharma and Chopra 1976; Babu 1977). Subsequent to characterization of the viable $w g^{1}$ allele, large-scale genetic screens performed by Eric Wieschaus, Christiane Nusslein-Volhard, and colleagues yielded embryonic lethal,

Editors: Roel Nusse, Xi He, and Renee van Amerongen

Additional Perspectives on Wnt Signaling available at www.cshperspectives.org

Copyright (C) 2012 Cold Spring Harbor Laboratory Press; all rights reserved; doi: 10.1101/cshperspect.a007930

Cite this article as Cold Spring Harb Perspect Biol 2012;4:a007930 
S. Swarup and E.M. Verheyen

Table 1. Comparison of Wnt genes between Drosophila and vertebrates

\begin{tabular}{|c|c|c|c|c|c|c|c|}
\hline \multicolumn{8}{|c|}{ Structural homology between Drosophila and vertebrate Wnt genes } \\
\hline $\begin{array}{c}\text { Drosophila } \\
\text { genes }\end{array}$ & $\begin{array}{l}\text { Dwnt-1 or } \\
\text { wingless }\end{array}$ & Dwnt-2 & Dwnt-5 & Dwnt-4 & Dwnt-6 & $\begin{array}{r}\text { Dwnt }-8 \text { or } \\
\text { Dwnt }-D\end{array}$ & Dwnt-10 \\
\hline $\begin{array}{l}\text { Vertebrate } \\
\text { homologs }\end{array}$ & Wnt-1 & Wnt-7 & Wnt-5 & $\begin{array}{l}\text { Wnt-9 } \\
\text { Wnt-14 } \\
\text { Wnt-15 }\end{array}$ & Wnt-6 & Wnt-8 & Wnt-10 \\
\hline
\end{tabular}

loss-of-function alleles of $w g$ (Nusslein-Volhard and Wieschaus 1980; Nusslein-Volhard et al. 1984). In the years that followed, the $w g$ gene was cloned (Baker 1987; Cabrera et al. 1987; Rijsewijk et al. 1987), and through the use of conditional mutants, mosaics analyses, and ectopic expression, it was shown to have important roles at several stages of development in multiple tissues, including the embryonic ectoderm (Baker 1988a; Bejsovec and Martinez Arias 1991; Dougan and DiNardo 1992; Bejsovec and Wieschaus 1993), head (Schmidt-Ott and Technau 1992), midgut (Immerglück et al. 1990; Reuter et al. 1990; Thuringer and Bienz 1993; Bienz 1994), wing disc (Simcox et al. 1989; Cohen 1990; Cohen et al. 1993; Phillips and Whittle 1993; Williams et al. 1993), and leg disc (Baker 1988b; Campbell et al. 1993; Couso et al. 1993). Moreover, through genetic and biochemical analyses performed predominantly in Drosophila over the years, the molecular mechanism of canonical Wnt or Wg signaling has emerged. In the absence of the Wnt/Wg ligand, cytoplasmic levels of $\beta$-catenin/Armadillo (Arm), the transcriptional effector of the pathway, are kept low through its constitutive degradation by a protein destruction complex composed of Axin, APC, GSK3/Zw3, and CK1. As a result, Wnt/Wg-regulated genes are kept off by the DNA-binding transcription factor T-cell factor (Tcf) with the aid of other transcriptional corepressors. Binding of the Wnt/Wg ligand to its coreceptors, Frizzled2 (Fz2) and LRP/Arrow (Arr), initiates a sequence of cytoplasmic events that leads to the Dishevelled (Dsh) - mediated inactivation of the protein destruction complex, thereby allowing stabilized $\beta$-catenin/Arm to translocate to the nucleus, where it binds Tcf to direct the activation of Wnt/ Wg-target genes (for review, see Bejsovec 2006).
In this article, we discuss the role of the $\mathrm{Wg}$ molecule as an organizing center during embryonic segmentation and patterning of the wing disc, because these are now considered the classic systems for demonstrating different aspects of Wg signaling.

\section{FUNCTION OF WINGLESS SIGNALING IN THE EMBRYO}

During Drosophila embryogenesis, a hierarchy of maternal and zygotic (gap, pair-rule, and segment polarity) genes progressively subdivides the embryonic syncytium into transverse regions that determine the anterior/posterior axis (for review, see Ingham and Martinez Arias 1992; St. Johnston and Nuesslein-Volhard 1992). The cellular blastoderm is formed during stage 14 of embryogenesis and coincides with the division of the anterior/posterior axis into segmental units as directed by the segment polarity genes $w g$ and hedgehog $(h h)$ (for review, see Perrimon 1994). These segment polarity genes interact with one another to define the segment boundaries and intrasegmental pattern of the embryo (Fig. 1). At the end of embryogenesis, the outcome of the segmentation and patterning events is a larva characterized on the ventral epidermis by an alternating pattern of protrusions called denticles that are separated by regions of naked cuticle ( for review, see Martinez-Arias 1993). We here describe the mechanism through which Wg signaling establishes and patterns each segment to generate this stereotypical arrangement of denticles and naked epidermal cuticle. This process can be divided into four successive events: establishment of the organizer, asymmetric signaling from the organizer, subdivision of each segment into 

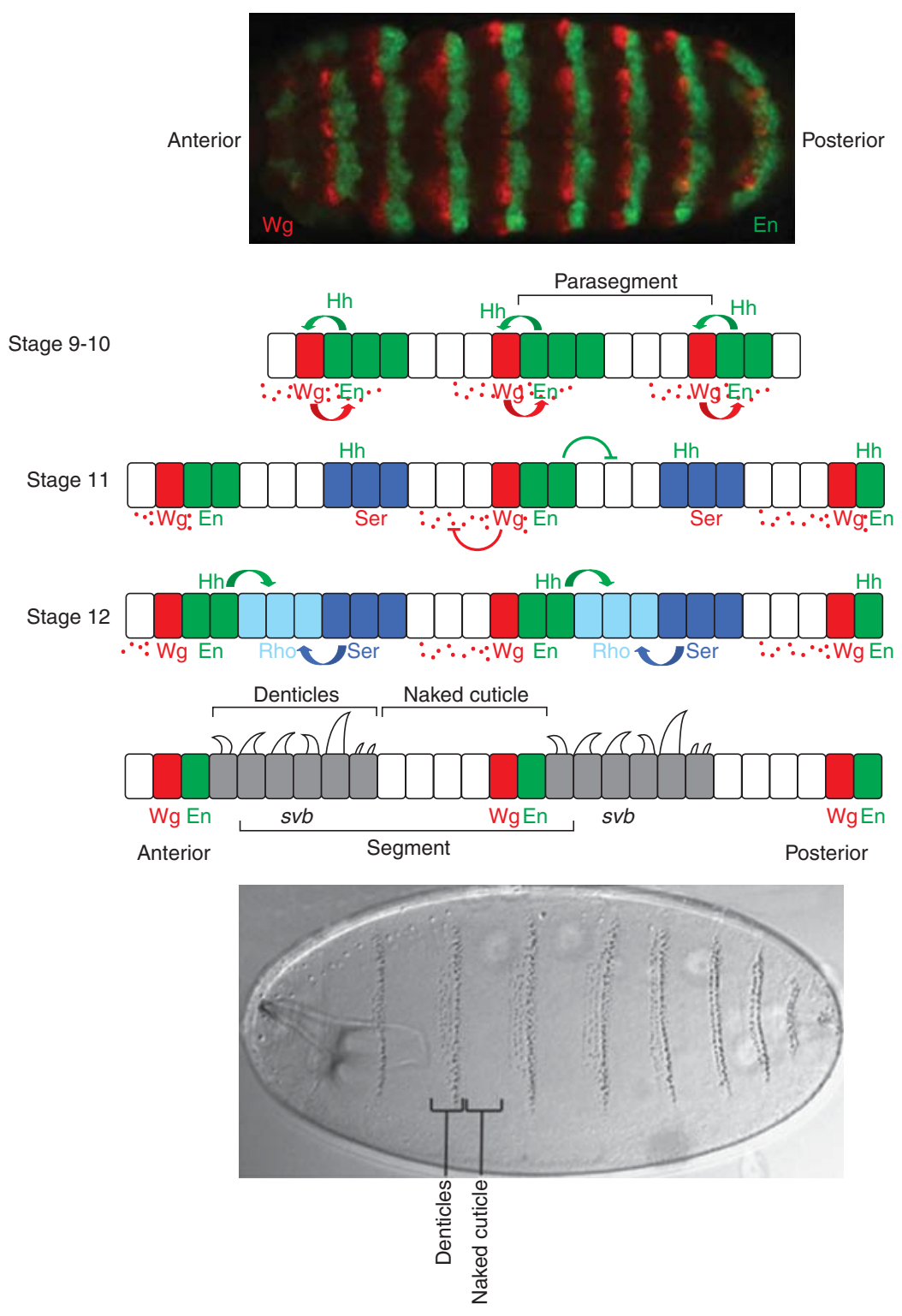

Figure 1. Wingless-regulated patterning of the Drosophila embryonic epidermis. The interplay between the Wg and Hh signaling pathways initially establishes the parasegment boundaries and subsequently directs the intrasegmental pattern to establish the stereotypical arrangement of denticles and naked cuticle at the end of embryogenesis (see text for details). The embryo is positioned with its anterior end to the left. (Top panel courtesy of L.R. Braid.)

signaling domains, and cell fate specification by the signaling domains (Fig. 1).

The expression of $w g$ and $h h$ is initiated by the pair-rule genes in adjacent, non-overlapping domains during stage 9-10 of embryogenesis, and subsequently, they reciprocally regu- late each other to stabilize their expression (Fig. 1) (for review, see DiNardo et al. 1994). Wg protein that is transcribed and secreted from an anterior row of cells maintains the expression of a transcription factor, engrailed (en), in adjoining, posterior cells. The En-expressing 
cells, in turn, transcribe and secrete the Hh ligand, which reciprocally maintains Wg expression in the neighboring, anterior cells (DiNardo et al. 1988; Martinez Arias et al. 1988; Hidalgo and Ingham 1990; Bejsovec and Martinez Arias 1991). The interface between these two adjacent domains defines the parasegment boundary or organizer, with en/hh transcribed at the anterior and $w g$ at the posterior end of each parasegment, respectively (Baker 1987; Lee et al. 1992; Mohler and Vani 1992).

Initially, after the parasegment boundary is established, the distribution of the Wg ligand is bidirectional and triggers a response through its signaling cascade at equivalent levels in both the anterior and posterior directions (Riggleman et al. 1990; Peifer et al. 1994). However, during stage 11, the distribution of extracellular $\mathrm{Wg}$ is only graded anterior to the Wg-expressing domain and is completely lost in the posterior direction (Fig. 1) (van den Heuvel et al. 1989; Gonzalez et al. 1991). This asymmetry in Wg distribution and correspondingly signaling activity is a result of its rapid endocytosis and degradation in cells posterior from which it is secreted, in a process that is promoted by $\mathrm{Hh}$ signaling (Sanson et al. 1999; Dubois et al. 2001). Interestingly, Wg signaling, in turn, attenuates $\mathrm{Hh}$ signaling anterior to the En/Hhexpressing cells, thereby allowing its activity in only the posterior direction (Gritzan et al. 1999). Thus, a pattern of polarized signaling of $\mathrm{Wg}$ and $\mathrm{Hh}$ at the parasegment boundary is formed that can direct cells on either side to follow distinct developmental programs.

The $w g$ - and en/hh-expressing domains initially established successively specify two additional domains within each parasegment that are defined by the expression of Serrate (Ser) (a ligand for the Notch pathway) (Wiellette et al. 1999) and rhomboid (rho) (promotes EGFR signaling) (Golembo et al. 1996). Wg and Hh signaling antagonize the posterior and anterior boundaries, respectively, of Ser expression, thereby restricting its domain to the center of each parasegment (Alexandre et al. 1999; Gritzan et al. 1999; Sanson et al. 1999). rho expression is repressed by Wg signaling and is positively reinforced through a combination of $\mathrm{Hh}$ and Ser signaling, thereby establishing its domain immediately posterior to the en/hh domain (Alexandre et al. 1999). During stage 12, each parasegment on the ventral epidermis is divided into four domains that express specific genes that are responsible for the intraparasegmental patterning of the embryo. This period also coincides with the formation of a segmental groove at the posterior edge of each $e n / h h$ domain and defines the segment boundary (Fig. 1).

The four signaling domains established within each segment control the binary decision between specification of naked cuticle or denticle cell fates. The outcome between these two choices is dependent on the expression of a transcription factor encoded by the shaven baby $(s v b)$ gene, which is necessary and sufficient to direct denticle formation cell autonomously (Payre et al. 1999). The expression of $s v b$ is inhibited in cells that specify naked cuticle, whereas cells that make denticles express $s v b$. Wg signaling specifies naked cuticle (Bejsovec and Martinez Arias 1991; Noordermeer et al. 1992; Lawrence et al. 1996) by repressing the expression of $s v b$ (Payre et al. 1999). Due to the asymmetric distribution of $\mathrm{Wg}$, the repression of $s v b$ is asymmetric and results in one row of cells posterior to, and four rows of cells anterior to the Wg domain that produce naked cuticle (O'Keefe et al. 1997; Szuts et al. 1997), across the posterior half of each segment. The six rows toward the anterior half of each segment do not receive the $\mathrm{Wg}$ ligand but instead transduce the EGFR signal that promotes $s v b$ expression (Payre et al. 1999), thus resulting in the generation of denticles that vary in shape, size, and polarity. The outcome of the patterning events mediated by regulated cell signaling is a repeated mosaic of denticle belts and naked cuticle on the ventral epidermis of the embryo, composed of segments $11-12$ cells wide along the anterior/posterior axis and 36-40 cells wide along the dorsal/ventral axis (Fig. 1). In $w g$ loss-of-function mutants, $s v b$ expression is not repressed and naked cuticle is not specified, resulting in the excess specification of denticles. Conversely, when $w g$ is ectopically expressed, excess naked cuticle is produced with 
a reduction or loss of denticles (Fig. 2) (Payre et al. 1999).

Components of the Wg signal transduction cascade were initially identified in large-scale genetic screens designed by Eric Wieschaus and Christiane Nusslein-Volhard to isolate zygotic mutations that disrupt the Drosophila embryonic cuticle. These screens yielded numerous genes that displayed segment polarity phenotypes when mutated, including both positive and negative regulators of the $\mathrm{Wg}$ pathway (Nusslein-Volhard and Wieschaus 1980; Jürgens et al. 1984; Nusslein-Volhard et al. 1984; Wieschaus et al. 1984). Mutations in arm and arr resemble the $w g$ loss-of-function phenotype to specify excess denticles, whereas naked, an inhibitor of the pathway, was also isolated and displays a $w g$ gain-of-function phenotype with an excess specification of naked cuticle. Although the aforementioned genetic screens led

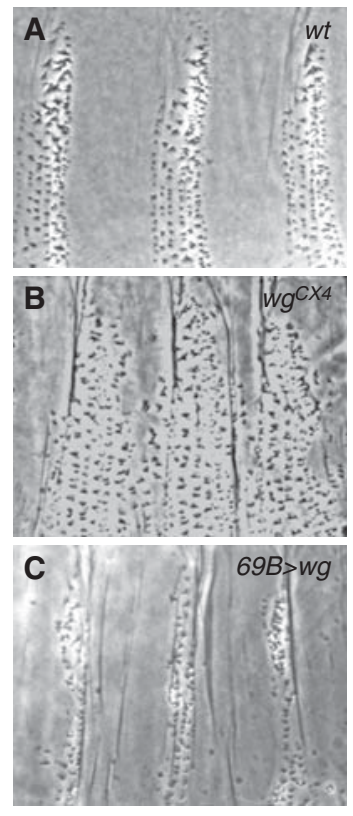

Figure 2. wingless mutant phenotypes of the Drosophila embryonic epidermis. (A) In a wild type (wt) embryo, $w g$ expression in segmental stripes generates regions of naked cuticle that are intercalated by regions of denticles. (B) A $w g^{C X 4}$ mutant embryo results in an excess specification of denticles, whereas (C) the overexpression of $w g$ (69B-Gal4>UAS-wg) results in the excess specification of naked cuticle. to the characterization of several downstream components, they did not yield the entire set of genes involved in the $\mathrm{Wg}$ pathway. This is because some genes are maternally contributed and zygotic mutants thus retain sufficient maternal product to pattern a relatively normal embryo. This problem was circumvented through the induction of mitotic recombination in the germline, thereby eliminating maternally contributed gene product, and this technique was used to genetically screen the $\mathrm{X}$-chromosome for maternal effect mutations that disrupt patterning of the embryonic cuticle (Perrimon et al. 1989). arm was reisolated in this screen along with additional new components of the pathway-dsh, porcupine, and $z w 3$.

Traditional loss-of-function epistasis experiments rely on evaluating phenotypes in double-mutant combinations, thus allowing one to determine which of the two distinct phenotypes "overrides" or is epistatic to the other. This information allows the ordering of genes within a pathway, because the gene that acts most downstream will generally be epistatic to those acting upstream. However, the similar phenotypes of many of the first identified patterning genes precluded such analyses. To overcome this limitation, numerous studies were performed using combinations of gain- and loss-of-function fly strains, which provided distinct phenotypes (e.g., excess naked cuticle due to gain of $w g$ compared with excess denticles in an arm mutant). These studies allowed the relationships between the early pathway components to be defined and have continued to serve as a template for characterizing and positioning novel pathway members. Remarkably, already in 1994, the minimal components of Wg signaling were known and ordered into a rudimentary pathway leading from $w g$ to $d s h, s g g, a r m$, and into the nucleus to regulate en expression (Noordermeer et al. 1994; Siegfried et al. 1994). Thus, through the implementation of genetic screens, several components of the $\mathrm{Wg}$ pathway were first defined based on their ability to disrupt embryonic patterning, and the order of these genes in the pathway was deduced through genetic epistasis. The genetic and molecular characterization of these components formed the 
S. Swarup and E.M. Verheyen

foundation of our current understanding of Wnt/Wg signaling.

\section{FUNCTION OF WINGLESS SIGNALING IN THE WING IMAGINAL DISC}

Like the embryonic ectoderm, the wing imaginal disc represents another tissue for which the function of Wg signaling has been well elucidated. The wing disc is an epithelial sac that is composed of 20 cells when it is formed during embryonic development and then proliferates during the larval stages to generate a disc of $\sim 75,000$ cells in the late third larval instar. At this stage, the cells comprising the major elements of the wing primordium can be identified through the use of molecular markers, including the notum, hinge, blade, and margin (Fig. 3). wg is expressed in two ring-like domains in the hinge region, along the dorsal/ ventral compartment boundary dividing the wing blade, and in a broad band in the dorsal part of the disc. The inner ring-like domain frames the wing blade and gives rise to the hinge, whereas the dorsal/ventral boundary region forms the wing margin. The most dorsal part of the wing disc gives rise to the notum of the adult fly (Fig. 3).

As previously mentioned, the first $w g^{1} \mathrm{mu}-$ tant displayed a transformation of wing structures into thoracic notal structures (Sharma and Chopra 1976; Morata and Lawrence 1977). Conversely, misexpression of $w g$ at a specific time leads to the ectopic induction of wing structures in the notum ( $\mathrm{Ng}$ et al. 1996; Klein and Arias 1998a). These phenotypic analyses suggest that the $w g$ gene has a crucial function during the development of the wing disc to specify the region that eventually gives rise to the adult wing. During the second larval instar, $w g$ is expressed in the ventral region of the wing disc and specifies the wing field, while the EGFR ligand, vein, is expressed in the dorsal region of the wing disc to specify the notum (Simcox et al. 1996; Wang et al. 2000). The loss of vein during the second larval instar results in the loss of all notal structures (Simcox et al. 1996; Wang et al. 2000), whereas the misexpression of vein in the wing field prevents wing formation and produces ectopic notum structures (Baonza et al. 2000; Wang et al. 2000), phenotypes that are complementary to those of $w g$. The expression of vein is restricted to the dorsal region of the wing disc by the suppressive influence of $\mathrm{Wg}$ signaling in the ventral region (Baonza et al. 2000). Conversely, $w g$ expression is antagonized in the dorsal region of the disc by EGFR signaling to limit its expression to only the ventral region (Baonza et al. 2000; Wang et al. 2000). This mutual antagonism between the $\mathrm{Wg}$ and EGFR pathways segregates the early wing disc into notum and wing regions and provides an explanation for the wing-to-notum transformation that occurs in the absence of $w g$ function.

Multiple pathways contribute to the refinement of the $w g$ expression pattern as larval

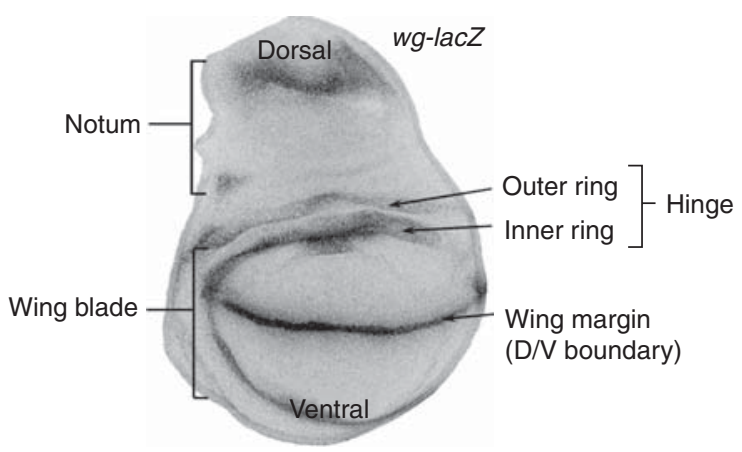

Figure 3. wingless expression in the wing imaginal disc. $w g$ is expressed at the dorsal/ventral boundary, in two concentric rings and in a broad stripe in the dorsal region of a third instar wing disc. The expression of $w g$ in the disc regulates the patterning of the wing margin, blade, hinge, and notum of the adult fly. 
development proceeds. During the late second instar, the expression of the selector gene apterous ( $a p$ ) overlaps ventrally with that of $w g$, to divide the developing wing field into two regions (Williams et al. 1993; Ng et al. 1996). The Notch pathway ligands, Serrate and Delta, are transcribed at the Ap-expression boundary (dorsal/ventral boundary) and lead to the induction of downstream genes that are ultimately required for the establishment of the distal wing fates, including the wing blade and margin (Williams et al. 1994; Klein and Arias 1998b; Milan and Cohen 2000). Subdivision of the wing field is initiated by the Notch-induced expression of vestigial $(\mathrm{vg})$ from its boundary enhancer $\left(v g^{B E}\right)$. Vg specifies the wing blade and collaborates with Notch signaling to induce the expression of $w g$ along the dorsal/ventral boundary (Kim et al. 1996; Klein and Arias 1998a, 1999). The Wg ligand is secreted from cells at the dorsal/ventral boundary and subsequently patterns the wing margin through activation of its target genes in a concentration-dependent manner (Couso et al. 1994; Neumann and Cohen 1997). After the establishment of the expression of $w g$ and $v g$ in the wing primordium, the wing blade begins to grow during the third instar. The expression of $v g$ is induced in cells of the wing blade that lie outside of the domain of Notch signaling through its quadrant enhancer $\left(v g^{Q E}\right)$ (Kim et al. 1996). This enhancer requires input from $\mathrm{Vg}$ itself, $\mathrm{Wg}$ (produced at the dorsal/ventral boundary), and Dpp (produced at the anterior/posterior boundary) for its activity.

Flies deficient for $w g$ do not develop wings, and mutant patches of cells that cannot respond to the Wg ligand are eliminated from the wing field in the wing disc (Chen and Struhl 1999). This suggests that there is an absolute requirement of Wg for growth of the wing. However, until recently it had been unclear whether cells of the wing disc required $\mathrm{Wg}$ signaling as an instructive signal or a permissive signal in order to proliferate. Early studies suggested that although the misexpression of $w g$ has mitogenic effects in the hinge region, it does not induce cell proliferation within the wing pouch but rather respecifies these cells to assume a wing margin fate (Neumann and Cohen 1996). It was subsequently shown that high levels of $\mathrm{Wg}$ at the presumptive wing margin, in fact, arrest the cell cycle (O'Brochta and Bryant 1985; Johnston and Edgar 1998). The ability of uniform expression of moderate levels of $\mathrm{Wg}$ to indeed promote growth throughout the presumptive wing region was shown only recently (BaenaLopez et al. 2009). In this study, the investigators propose a model in which Vg-expressing cells expand their numbers by inducing their neighboring, non-wing cells to express $\mathrm{Vg}$, provided these cells also receive the $\mathrm{Wg}$ signal, and thereby be recruited into the wing field through a feed-forward mechanism. In this scenario, $\mathrm{Wg}$ is proposed to act as a permissive signal to enable the recruitment of cells into the wing field to promote growth of the wing disc (Zecca and Struhl 2007a,b).

\section{DOES WINGLESS ALWAYS BEHAVE AS A MORPHOGEN?}

As a minimal definition, a "morphogen" is a molecule that diffuses away from a localized source to directly instruct cell identities in a concentration-dependent manner. For a secreted signaling ligand such as Wg to qualify as a morphogen, it must form a graded distribution away from its source, directly act on cells at a distance rather than indirectly through a relay mechanism, and induce the commitment of cells in the field, as a function of their distance from the morphogen source, to distinct developmental fates through the expression of different sets of genes.

Experiments performed in the wing disc suggest that Wg does indeed behave as a morphogen in this tissue. During the third larval instar, $\mathrm{Wg}$, secreted from cells at the dorsal/ ventral organizing boundary of the wing disc, diffuses away from its source on either side and acts directly on cells at long range to induce the nested expression of its target genes achaete (ac), distal-less (dll), and vestigial (vg) (Fig. 4) (Zecca et al. 1996; Neumann and Cohen 1997). The modulation of $\mathrm{Wg}$ signaling through clonal analyses or ectopic expression of arm and $d s h$, positive regulators of the pathway, shows 
S. Swarup and E.M. Verheyen

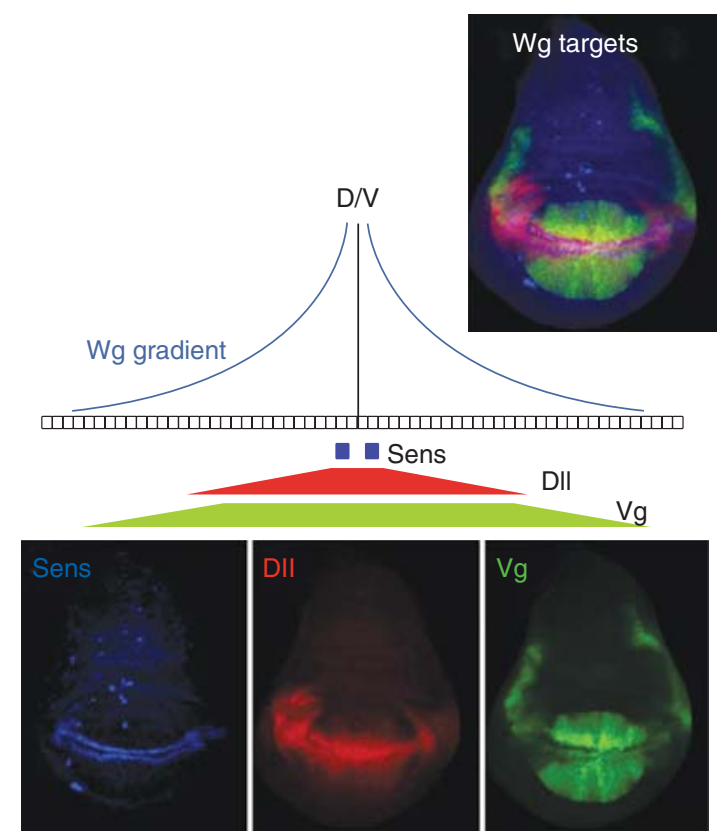

Figure 4. Morphogen gradient of Wingless. In the third instar wing disc, Wg is secreted from cells at the dorsal/ ventral boundary and forms a concentration gradient on either side to regulate the expression of its target genes, senseless (Sens, blue, high threshold), distalless (Dll, red, medium threshold), and vestigial (Vg, green, low threshold). The expression of distalless is graded throughout its domain, whereas vestigial expression is graded only at its edges. senseless, like achaete (discussed in the text), requires high levels of Wg signaling and is expressed only in cells abutting the dorsal/ventral boundary.

a cell-autonomous change in Wg-responsive target gene expression, regardless of the position of the cell from the source of the Wg signal (Zecca et al. 1996; Neumann and Cohen 1997). These data suggest that the $\mathrm{Wg}$ ligand acts directly on cells distant from its source, and the expression of target genes is not activated via a secondary or relay signal. In addition, in contrast to the wild type secreted $\mathrm{Wg}$, a membrane-tethered form of the ligand that is unable to diffuse away from its source, activates expression of target genes only in its immediate neighbors (Zecca et al. 1996; Neumann and Cohen 1997). Furthermore, through the use of a temperature-sensitive allele, $w g^{t s}$, it has been shown that the level of $\mathrm{Wg}$ activity minimally required to activate expression of $a c$, a highthreshold target gene; dll, a medium-threshold target gene; and $v g$, a low-threshold target gene; is progressively lower. Because the activity of $\mathrm{Wg}^{\text {ts }}$ is decreased (by raising the temperature), the expression of $a c$ is lost at a temperature at which dll expression is retained, and as the temperature is further increased, the expression of $d l l$ is lost with no effect on $v g$ expression. In both cases, there is a concomitant reduction of the expression domains of target genes toward the source of the ligand as the activity level of $\mathrm{Wg}$ is reduced. Consistent with this result, low levels of ectopically expressed $\mathrm{Wg}$ are able to activate dll but not ac (Neumann and Cohen 1997). These data suggest that Wg activates different target genes in a concentration-dependent manner and defines their expression domains through different activation thresholds. This does not exclude the possibility that activation of target genes requires other permissive signals, but it does argue that the level of the $\mathrm{Wg}$ ligand is the instructive signal. Lastly, the extracellular concentration gradient of the Wg protein can be detected up to 10 cell diameters away from the secreting cells at the dorsal/ventral 
boundary and represents direct evidence that Wg acts as a morphogen in the wing disc (Strigini and Cohen 2000).

Wingless has been proposed to behave as a morphogen additionally in the embryonic epidermis and midgut, but whether it actually does so in these developmental contexts is unclear. In the embryonic ectoderm, Wg secreted from the parasegment boundary adopts a graded distribution in the anterior direction up to four cells away to specify naked cuticle (Bejsovec and Martinez Arias 1991). Although in this context Wg fulfills the criteria of having a localized source, forming a gradient, and acting at long range, there is no evidence for a concentration-dependent induction of target genes. In fact, the epidermal phenotype of $w g$ mutant embryos can be rescued through the ubiquitous expression of a $w g$ transgene (Sampedro et al. 1993), suggesting that Wg acts in a gradientindependent manner in this tissue. Additionally, in a wg mutant background, the overexpression of a membrane-tethered form of $\mathrm{Wg}$ from cells that normally express the ligand can recapitulate the normal range of signaling, confirming that the restricted diffusion of $\mathrm{Wg}$ is dispensable for patterning of the embryonic cuticle (Pfeiffer et al. 2000). In the embryonic midgut, $w g$ is expressed in the visceral mesoderm and directs the neighboring endoderm to differentiate into two different cell types: large, flat cells require high levels of signaling and develop immediately adjacent to the $\mathrm{Wg}$ source, whereas copper cells develop further away as their differentiation is repressed at high levels of $\mathrm{Wg}$ signaling. As the level of $\mathrm{Wg}$ is modified, there is a concomitant change in the domains of the large, flat cells and copper cells. Additionally, the labial gene is expressed in a graded manner in the region of copper cells, consistent with the proposed concentration-dependent effect of Wg. However, the effects of Wg have not been shown to be direct in this tissue (Hoppler and Bienz 1995). Thus, in the embryonic ectoderm and midgut, it is possible that $\mathrm{Wg}$ signaling does not define the pattern of the response but, rather, stabilizes patterns of gene expression that have been specified through other mechanisms.

\section{ENDOCYTOSIS: SHAPING THE WINGLESS GRADIENT}

Secreted Wg is theoretically capable of passive diffusion at a relatively fast rate in all directions over a long range. However, the graded distribution of $\mathrm{Wg}$ forms at a slower rate over a shorter range than that predicted through free diffusion and directionally along the epithelial surface. Moreover, the contrasting range of its activity during embryogenesis (4 cell diameters) (Bejsovec and Martinez Arias 1991; Pfeiffer et al. 2002) versus development of the wing disc (10 cell diameters) (Strigini and Cohen 2000) suggests that the movement of the $\mathrm{Wg}$ ligand in the extracellular environment is regulated. Recent experimental and theoretical studies favor a model in which the $\mathrm{Wg}$ gradient is formed and maintained through the combined effects of restricted diffusion and endocytosis. Once secreted, the Wg molecule undergoes restricted diffusion, as opposed to free diffusion, because of its interactions with lipid-based transport proteins, receptors on the surface of membranes, and heparan sulfate proteoglycans of the extracellular matrix (Lin and Perrimon 1999; Baeg et al. 2001, 2004; Lecourtois et al. 2001; Bornemann et al. 2004; Kirkpatrick et al. 2004; Han et al. 2005; Panakova et al. 2005; Piddini et al. 2005; Katanaev et al. 2008; Mulligan et al. 2012). In addition, the repeated vesicle-mediated endocytosis and resecretion of Wg, in a process called "planar transcytosis," and the endocytosis-mediated degradation of Wg also contribute to the formation of the Wg gradient, both extracellularly and intracellularly. We here review the evidence for the endocytosis-mediated regulation of the $\mathrm{Wg}$ gradient in the Drosophila embryo and wing disc.

At the subcellular level, Wg protein can be detected not only in the extracellular environment, but also within intracellular vesicles and multivesicular bodies in non-secreting cells. This clearly indicates that the Wg ligand is internalized and suggests that its gradient is regulated through endocytosis. The endocytic regulation of the $\mathrm{Wg}$ gradient has been investigated using mutations in components of the endocytic machinery. The formation of the $\mathrm{Wg}$ 
gradient in the embryo is a result of asymmetric endocytosis and trafficking to lysosomes, and perhaps transcytosis. As previously described, during embryogenesis, the initially symmetrical $\mathrm{Wg}$ gradient becomes asymmetrical due to increased Wg degradation in cells posterior to its source (Fig. 1) (Sanson et al. 1999; Dubois et al. 2001). Evidence for this model comes from experiments performed using an HRP (horse radish peroxidase) $-\mathrm{Wg}$ fusion protein expressed under the control of the endogenous $w g$ promoter. Unlike the $\mathrm{Wg}$ portion of the fusion protein, the HRP moiety is stable throughout the endocytic pathway and thus serves as a tool to monitor Wg degradation in vivo. In cells anterior to the HRP-Wg source, both HRP and $\mathrm{Wg}$ can be detected in intracellular vesicles (presumed to be early endosomes), whereas only HRP can be detected in vesicles (presumably late endosomes) in cells posterior to the source. Moreover, posterior to the Wg source, many more HRP-positive intracellular compartments can be detected that extend beyond the $\mathrm{Wg}$ protein gradient, confirming that the degradation of Wg through the endocytic pathway limits the range of its gradient (Dubois et al. 2001). In deep orange (dor) mutants that have impaired trafficking to the lysosome, $\mathrm{Wg}$ accumulates in multivesicular bodies (Piddini et al. 2005). Lastly, in clathrin heavy chain (chc) mutants that cannot initiate clathrin-mediated endocytosis, the Wg gradient extends in the posterior direction, suggesting that clathrin is normally required for establishing and maintaining the asymmetric distribution of $\mathrm{Wg}$ (Desbordes et al. 2005). However, there is also contradictory evidence that suggests a role for endocytosis in Wg dispersal in the embryo. In temperature-sensitive shibire ${ }^{t s}$ (which encodes Dynamin, an essential component of both clathrin and caveolin-mediated endocytosis) mutant embryos that are shifted to the restrictive temperature, the $\mathrm{Wg}$ gradient is reduced to a narrow range around the Wg-expressing cells (Moline et al. 1999). This narrowed Wg gradient in shibire $e^{t s}$ embryos is not due to any effect on the secretion of the $\mathrm{Wg}$ ligand or its stability in non-secreting cells but, rather, can be attributed to an effect on the transport of the $\mathrm{Wg}$ ligand extracellularly (Moline et al. 1999). The visualization of the trafficking of a Wg-GFP fusion protein in live embryos reveals that $\mathrm{Wg}$ can be internalized and recycled back to the cell surface (Pfeiffer et al. 2000), providing additional evidence of a role for transcytosis in $\mathrm{Wg}$ distribution.

In the wing disc, the $\mathrm{Wg}$ protein can indeed traverse a shibire mutant clone and can be detected extracellularly both proximal and distal to the clone (Strigini and Cohen 2000). This suggests that in the wing disc, the Wg ligand once secreted is able to spread to non-expressing cells in the absence of endocytic trafficking. In fact, the inhibition of endocytosis leads to an extension of the $\mathrm{Wg}$ gradient, indicating that endocytosis serves to down-regulate the levels of $\mathrm{Wg}$ in the wing disc (Strigini and Cohen 2000; Piddini et al. 2005). In accordance with this hypothesis, more extracellular $\mathrm{Wg}$ is present within the shibire mutant clone than outside the clone (Strigini and Cohen 2000).

Notably, the majority of evidence from the embryonic epidermis and wing disc support different models of Wg transport and stability that contribute to the Wg gradient. Further studies need to be performed to resolve which mechanism of $\mathrm{Wg}$ distribution is predominant under physiological conditions.

\section{WINGLESS-RESPONSIVE TARGET GENES: ACTIVATION VERSUS REPRESSION}

Although the mechanistic details through which cell signaling pathways ultimately regulate gene expression to control the development of metazoans may differ, they all share at least three common, conserved features: default repression, activator insufficiency, and cooperative activation (for review, see Barolo et al. 2002; Affolter et al. 2008). Developmental signaling pathways regulate gene expression by a switch mechanism, whereby from an actively repressed state in the absence of the signal, genes are transcribed in the presence of the signal. The phenomenon of inhibition of gene transcription in the absence of signaling is referred to as "default repression." "Activator insufficiency" and "cooperative activation" refer to the 
inability of a signaling pathway to activate the same and complete set of target genes in all developmental contexts. The combination of these three features allows a signaling pathway to robustly activate specific target gene expression in response to the signal, in a context-dependent manner, while preventing target gene expression in the absence of the signal (for review, see Barolo et al. 2002; Affolter et al. 2008).

In the case of Wg signaling, default repression is exerted on the same pathway response element using the same signal-regulated transcription factor, Tcf, as is used in the presence of active signaling. In the absence of signaling, Tcf binds Wg-responsive elements within target genes along with other corepressors to suppress gene expression. When the Wg ligand is present, the same transcription factor Tcf binds Arm and recruits other coactivators to direct the expression of target genes (Brunner et al. 1997; van de Wetering et al. 1997; Lawrence et al. 2000; Schweizer et al. 2003). However, it has recently become apparent that target genes of the pathway are not only directly activated by the Wg signal, but can be directly repressed as well. Direct repression implies that following signaling, a gene's transcription is repressed without the intermediate transcriptional induction of a repressor. Signal-induced gene repression conflicts with the principle of default repression. If gene transcription is silenced through default repression before pathway activity, there is no opportunity to repress gene transcription following signaling. Indeed, for Wg signaling to repress transcription of a gene, default repression of the gene would have to be circumvented before signaling with a switch to a default activation state. Conceptually, the reversal of this feature would allow signaling pathways to robustly repress target gene expression in the presence of the signal, in a context-dependent manner, while activating the target gene in its absence.

Several target genes including $f z 2, s v b$, rho, Ultrabithorax $(U b x)$, stripe $(s r)$, and $d p p$ have been proposed to be directly repressed by $\mathrm{Wg}$ signaling. However, in the cases of $f z 2, s v b$, and rho (Cadigan et al. 1998; Payre et al. 1999; Sanson et al. 1999), the molecular studies address- ing the cis and/or trans regulatory elements involved in transcriptional repression are lacking, while for $U b x$, it is thought that repression of gene transcription occurs indirectly and is therefore a secondary effect of $\mathrm{Wg}$ signaling (Waltzer et al. 2001). Only in the cases of $s r$ in the embryonic epidermis (Piepenburg et al. 2000) and $d p p$ in the leg imaginal disc (Theisen et al. 2007), has it been convincingly shown that these genes are, in fact, repressed by Tcf in the presence of signaling. Both of these genes contain functional Tcf-binding sites in their response elements that are required for repression, and mutation of these sites results in a failure of Wg-mediated gene silencing.

In a recent study, several genes that are repressed by Wg signaling were identified from cultured Drosophila hemocytic cells. Surprisingly, the characterization of the cis regulatory element of one of these genes, Ugt36Bc, revealed that the Tcf recognition site is markedly different from a typical consensus Tcf binding site. Furthermore, the novel Tcf binding sites were not only required for $\mathrm{Wg}$-induced repression, but were also essential for the default transcription of Ugt36Bc in the absence of signaling (Blauwkamp et al. 2008). This suggests that the nature of the Tcf binding site within the $\mathrm{Wg}$-responsive element can determine the nature of the transcriptional output, both in the absence and presence of signaling, and is one possible mechanism through which Wg signaling can switch from activation to repression of certain genes.

\section{CONCLUDING REMARKS}

Over the last 30 years, the use of genetic analyses in Drosophila has elucidated both the function, in various contexts of development, and the molecular mechanism of the Wg pathway. In addition, the study of $\mathrm{Wnt} / \mathrm{Wg}$ proteins first illustrated the relationship between normal development and oncogenesis. Owing to the vast array of genetic techniques available in Drosophila and its high degree of conservation, no doubt, novel components and Wg-regulated processes identified in future studies using this model system are likely to be directly applicable to vertebrate development. 


\section{ACKNOWLEDGMENTS}

We gratefully acknowledge support from a Natural Sciences and Engineering Research Council of Canada Doctoral Research Award (to S.S.) and a Canadian Institutes for Health Research operating grant (to E.M.V.). We apologize to those colleagues whose work was not cited because of length restrictions.

\section{REFERENCES}

Affolter M, Pyrowolakis G, Weiss A, Basler K. 2008. Signalinduced repression: The exception or the rule in developmental signaling? Dev Cell 15: 11-22.

Alexandre C, Lecourtois M, Vincent J. 1999. Wingless and Hedgehog pattern Drosophila denticle belts by regulating the production of short-range signals. Development 126: 5689-5698.

Babu P. 1977. Early developmental subdivisions of the wing disk in Drosophila. Mol Gen Genet 151: 289-294.

Baeg GH, Lin X, Khare N, Baumgartner S, Perrimon N. 2001. Heparan sulfate proteoglycans are critical for the organization of the extracellular distribution of Wingless. Development 128: 87-94.

Baeg GH, Selva EM, Goodman RM, Dasgupta R, Perrimon N. 2004. The Wingless morphogen gradient is established by the cooperative action of Frizzled and Heparan Sulfate Proteoglycan receptors. Dev Biol 276: 89-100.

Baena-Lopez LA, Franch-Marro X, Vincent JP. 2009. Wingless promotes proliferative growth in a gradient-independent manner. Sci Signal 2: ra60.

Baker NE. 1987. Molecular cloning of sequences from wingless, a segment polarity gene in Drosophila: The spatial distribution of a transcript in embryos. EMBO $J$ 6: 1765-1773.

Baker NE. 1988a. Embryonic and imaginal requirements for wingless, a segment-polarity gene in Drosophila. Dev Biol 125: $96-108$.

Baker NE. 1988b. Transcription of the segment-polarity gene wingless in the imaginal discs of Drosophila, and the phenotype of a pupal-lethal $w g$ mutation. Development 102: 489-497.

Baonza A, Roch F, Martin-Blanco E. 2000. DER signaling restricts the boundaries of the wing field during Drosophila development. Proc Natl Acad Sci 97: 7331-7335.

Barolo S, Stone T, Bang AG, Posakony JW. 2002. Default repression and Notch signaling: Hairless acts as an adaptor to recruit the corepressors Groucho and dCtBP to Suppressor of Hairless. Genes Dev 16: 1964-1976.

Bejsovec A. 2006. Flying at the head of the pack: Wnt biology in Drosophila. Oncogene 25: 7442-7449.

Bejsovec A, Martinez Arias A. 1991. Roles of wingless in patterning the larval epidermis of Drosophila. Development 113: 471-485.

Bejsovec A, Wieschaus E. 1993. Segment polarity gene interactions modulate epidermal patterning in Drosophila embryos. Development 119: 501-517.
Bienz M. 1994. Homeotic genes and positional signalling in the Drosophila viscera. Trends Genet 10: 22-26.

Blauwkamp TA, Chang MV, Cadigan KM. 2008. Novel TCFbinding sites specify transcriptional repression by Wnt signalling. EMBO J 27: 1436-1446.

Bornemann DJ, Duncan JE, Staatz W, Selleck S, Warrior R. 2004. Abrogation of heparan sulfate synthesis in Drosophila disrupts the Wingless, Hedgehog and Decapentaplegic signaling pathways. Development 131: 1927-1938.

Brunner E, Peter O, Schweizer L, Basler K. 1997. pangolin encodes a Lef-1 homologue that acts downstream of Armadillo to transduce the Wingless signal in Drosophila. Nature 385: 829-833.

Cabrera CV, Alonso MC, Johnston P, Phillips RG, Lawrence PA. 1987. Phenocopies induced with antisense RNA identify the wingless gene. Cell 50: 659-663.

Cadigan KM, Fish MP, Rulifson EJ, Nusse R. 1998. Wingless repression of Drosophila frizzled 2 expression shapes the Wingless morphogen gradient in the wing. Cell 93: 767-777.

Campbell G, Weaver T, Tomlinson A. 1993. Axis specification in the developing Drosophila appendage: The role of wingless, decapentaplegic, and the homeobox gene aristaless. Cell 74: 1113-1123.

Chen CM, Struhl G. 1999. Wingless transduction by the Frizzled and Frizzled2 proteins of Drosophila. Development 126: 5441-5452.

Cohen SM. 1990. Specification of limb development in the Drosophila embryo by positional cues from segmentation genes. Nature 343: 173-177.

Cohen B, Simcox AA, Cohen SM. 1993. Allocation of the thoracic imaginal primordia in the Drosophila embryo. Development 117: 597-608.

Couso JP, Bate M, Martinez-Arias A. 1993. A wingless-dependent polar coordinate system in Drosophila imaginal discs. Science 259: 484-489.

Couso JP, Bishop SA, Martinez-Arias A. 1994. The wingless signalling pathway and the patterning of the wing margin in Drosophila. Development 120: 621-636.

Desbordes SC, Chandraratna D, Sanson B. 2005. A screen for genes regulating the Wingless gradient in Drosophila embryos. Genetics 170: 749-766.

DiNardo S, Sher E, Heemskerk-Jongens J, Kassis JA, O'Farrell PH. 1988. Two-tiered regulation of spatially patterned engrailed gene expression during Drosophila embryogenesis. Nature 332: 604-609.

DiNardo S, Heemskerk J, Dougan S, O’Farrell PH. 1994. The making of a maggot: Patterning the Drosophila embryonic epidermis. Curr Opin Genet Dev 4: 529-534.

Dougan S, DiNardo S. 1992. Drosophila wingless generates cell type diversity among engrailed expressing cells. $\mathrm{Na}$ ture 360: 347-350.

Dubois L, Lecourtois M, Alexandre C, Hirst E, Vincent JP. 2001. Regulated endocytic routing modulates Wingless signaling in Drosophila embryos. Cell 105: 613-624.

Golembo M, Raz E, Shilo BZ. 1996. The Drosophila embryonic midline is the site of Spitz processing, and induces activation of the EGF receptor in the ventral ectoderm. Development 122: 3363-3370.

Gonzalez F, Swales L, Bejsovec A, Skaer H, Martinez Arias A. 1991. Secretion and movement of Wingless protein in the 
epidermis of the Drosophila embryo. Mech Dev 35: 43-54.

Gritzan U, Hatini V, DiNardo S. 1999. Mutual antagonism between signals secreted by adjacent Wingless and Engrailed cells leads to specification of complementary regions of the Drosophila parasegment. Development 126: 4107-4115.

Han C, Yan D, Belenkaya TY, Lin X. 2005. Drosophila glypicans Dally and Dally-like shape the extracellular Wingless morphogen gradient in the wing disc. Development 132: 667-679.

Hidalgo A, Ingham P. 1990. Cell patterning in the Drosophila segment: Spatial regulation of the segment polarity gene patched. Development 110: 291-301.

Hoppler S, Bienz M. 1995. Two different thresholds of wingless signalling with distinct developmental consequences in the Drosophila midgut. EMBO J 14: 5016-5026.

Immerglück K, Lawrenc PA, Bienz M. 1990. Induction across germ layers in Drosophila mediated by a genetic cascade. Cell 62: 261-268.

Ingham PW, Martinez Arias A. 1992. Boundaries and fields in early embryos. Cell 68: 221-235.

Johnston LA, Edgar BA. 1998. Wingless and Notch regulate cell-cycle arrest in the developing Drosophila wing. $\mathrm{Na}$ ture 394: 82-84

Jürgens G, Wieschaus E, Nüsslein-Volhard C, Kluding H. 1984. Mutations affecting the pattern of the larval cuticle in Drosophila melanogaster ctII: Zygotic loci on the third chromosome. Rouxs Arch Dev Biol 193: 283-295.

Katanaev VL, Solis GP, Hausmann G, Buestorf S, Katanayeva N, Schrock Y, Stuermer CA, Basler K. 2008. Reggie-1/ flotillin-2 promotes secretion of the long-range signalling forms of Wingless and Hedgehog in Drosophila. EMBO J 27: $509-521$.

Kim J, Sebring A, Esch J, Kraus M, Vorwerk K, Magee J, Carroll S. 1996. Integration of positional signals and regulation of wing formation and identity by Drosophila vestigial gene. Nature 382: 133-138.

Kirkpatrick CA, Dimitroff BD, Rawson JM, Selleck SB. 2004. Spatial regulation of Wingless morphogen distribution and signaling by Dally-like protein. Dev Cell 7: 513-523.

Klein T, Arias AM. 1998a. Different spatial and temporal interactions between Notch, wingless, and vestigial specify proximal and distal pattern elements of the wing in Drosophila. Dev Biol 194: 196-212.

Klein T, Arias AM. 1998b. Interactions among Delta, Serrate and Fringe modulate Notch activity during Drosophila wing development. Development 125: 2951-2962.

Klein T, Arias AM. 1999. The vestigial gene product provides a molecular context for the interpretation of signals during the development of the wing in Drosophila. Development 126: 913-925.

Lawrence PA, Sanson B, Vincent JP. 1996. Compartments, wingless and engrailed: Patterning the ventral epidermis of Drosophila embryos. Development 122: 4095-4103.

Lawrence N, Dearden P, Hartley D, Roose J, Clevers H, Arias AM. 2000. dTcf antagonises Wingless signalling during the development and patterning of the wing in Drosophila. Int J Dev Biol 44: 749-756.
Lecourtois M, Alexandre C, Dubois L, Vincent JP. 2001. Wingless capture by Frizzled and Frizzled2 in Drosophila embryos. Dev Biol 235: 467-475.

Lee JJ, von Kessler DP, Parks S, Beachy PA. 1992. Secretion and localized transcription suggest a role in positional signaling for products of the segmentation gene hedgehog. Cell 71: 33-50.

Lin X, Perrimon N. 1999. Dally cooperates with Drosophila Frizzled 2 to transduce Wingless signalling. Nature 400: 281-284.

Martinez-Arias A. 1993. Development and patterning of the larval epidermis of Drosophila. In The development of Drosophila melanogaster (ed. M Bate, A Martinez-Arias), pp. 517-608. Cold Spring Harbor Laboratory Press, Cold Spring Harbor, NY.

Martinez Arias A, Baker NE, Ingham PW. 1988. Role of segment polarity genes in the definition and maintenance of cell states in the Drosophila embryo. Development 103: 157-170.

Milan M, Cohen SM. 2000. Temporal regulation of apterous activity during development of the Drosophila wing. Development 127: 3069-3078.

Mohler J, Vani K. 1992. Molecular organization and embryonic expression of the hedgehog gene involved in cell-cell communication in segmental patterning of Drosophila. Development 115: 957-971.

Moline MM, Southern C, Bejsovec A. 1999. Directionality of Wingless protein transport influences epidermal patterning in the Drosophila embryo. Development 126: 43754384.

Morata G, Lawrence PA. 1977. The development of wingless, a homeotic mutation of Drosophila. Dev Biol 56: 227240.

Mulligan K, Fuerer C, Ching W, Fish M, Willert K, Nusse R. 2012. Secreted Wingless-interacting molecule (Swim) promotes long-range signaling by maintaining Wingless solubility. Proc Natl Acad Sci 109: 370-377.

Neumann CJ, Cohen SM. 1996. Distinct mitogenic and cell fate specification functions of wingless in different regions of the wing. Development 122: 1781-1789.

Neumann CJ, Cohen SM. 1997. Long-range action of Wingless organizes the dorsal-ventral axis of the Drosophila wing. Development 124: 871-880.

Ng M, Diaz-Benjumea FJ, Vincent JP, Wu J, Cohen SM. 1996. Specification of the wing by localized expression of Wingless protein. Nature 381: 316-318.

Noordermeer J, Johnston P, Rijsewijk F, Nusse R, Lawrence P. 1992. The consequences of ubiquitous expression of the wingless gene in the Drosophila embryo. Development 116: $711-719$.

Noordermeer J, Klingensmith J, Perrimon N, Nusse R. 1994. dishevelled and armadillo act in the Wingless signalling pathway in Drosophila. Nature 367: 80-83.

Nusse R, Varmus HE. 1982. Many tumors induced by the mouse mammary tumor virus contain a provirus integrated in the same region of the host genome. Cell 31: 99-109.

Nusse R, van Ooyen A, Cox D, Fung YK, Varmus H. 1984. Mode of proviral activation of a putative mammary oncogene (int-1) on mouse chromosome 15. Nature 307: 131-136. 
Nusslein-Volhard C, Wieschaus E. 1980. Mutations affecting segment number and polarity in Drosophila. Nature 287: 795-801.

Nusslein-Volhard C, Wiechaus E, Kluding H. 1984. Mutations affecting the pattern of the larval cuticle in Drosophila melanogaster. I. Zygotic loci on the second chromosome. Rouxs Arch Dev Biol 193: 267-282.

O’Brochta DA, Bryant PJ. 1985. A zone of non-proliferating cells at a lineage restriction boundary in Drosophila. $\mathrm{Na}$ ture 313 138-141.

O’Keefe L, Dougan ST, Gabay L, Raz E, Shilo BZ, DiNardo S. 1997. Spitz and Wingless, emanating from distinct borders, cooperate to establish cell fate across the Engrailed domain in the Drosophila epidermis. Development 124: 4837-4845.

Panakova D, Sprong H, Marois E, Thiele C, Eaton S. 2005. Lipoprotein particles are required for Hedgehog and Wingless signalling. Nature 435: 58-65.

Payre F, Vincent A, Carreno S. 1999. ovo/svb integrates Wingless and DER pathways to control epidermis differentiation. Nature 400: 271-275.

Peifer M, Sweeton D, Casey M, Wieschaus E. 1994. wingless signal and Zeste-white 3 kinase trigger opposing changes in the intracellular distribution of Armadillo. Development 120: 369-380.

Perrimon N. 1994. The genetic basis of patterned baldness in Drosophila. Cell 76: 781-784.

Perrimon N, Engstrom L, Mahowald A. 1989. Zygotic lethals with specific maternal effect phenotypes in Drosophila melanogaster I loci on the X chromosome. Genetics 121: $333-352$.

Pfeiffer S, Alexandre C, Calleja M, Vincent JP. 2000. The progeny of wingless-expressing cells deliver the signal at a distance in Drosophila embryos. Curr Biol 10: 321-324.

Pfeiffer S, Ricardo S, Manneville JB, Alexandre C, Vincent JP. 2002. Producing cells retain and recycle Wingless in Drosophila embryos. Curr Biol 12: 957-962.

Phillips RG, Whittle JR. 1993. wingless expression mediates determination of peripheral nervous system elements in late stages of Drosophila wing disc development. Development 118: 427-438.

Piddini E, Marshall F, Dubois L, Hirst E, Vincent JP. 2005. Arrow (LRP6) and Frizzled2 cooperate to degrade Wingless in Drosophila imaginal discs. Development 132: 5479-5489.

Piepenburg O, Vorbruggen G, Jackle H. 2000. Drosophila segment borders result from unilateral repression of Hedgehog activity by Wingless signaling. Mol Cell 6: 203-209.

Reuter R, Panganiban GE, Hoffmann FM, Scott MP. 1990. Homeotic genes regulate the spatial expression of putative growth factors in the visceral mesoderm of Drosophila embryos. Development 110: 1031-1040.

Riggleman B, Schedl P, Wieschaus E. 1990. Spatial expression of the Drosophila segment polarity gene armadillo is posttranscriptionally regulated by wingless. Cell 63: 549-560.

Rijsewijk F, Schuermann M, Wagenaar E, Parren P, Weigel D, Nusse R. 1987. The Drosophila homolog of the mouse mammary oncogene int-1 is identical to the segment polarity gene wingless. Cell 50: 649-657.
Sampedro J, Johnston P, Lawrence PA. 1993. A role for wingless in the segmental gradient of Drosophila? Development 117: 677-687.

Sanson B, Alexandre C, Fascetti N, Vincent JP. 1999. Engrailed and Hedgehog make the range of Wingless asymmetric in Drosophila embryos. Cell 98: 207-216.

Schmidt-Ott U, Technau GM. 1992. Expression of en and $w g$ in the embryonic head and brain of Drosophila indicates a refolded band of seven segment remnants. Development 116: $111-125$.

Schweizer L, Nellen D, Basler K. 2003. Requirement for Pangolin/dTCF in Drosophila Wingless signaling. Proc Natl Acad Sci 100: 5846-5851.

Sharma RP, Chopra VL. 1976. Effect of the wingless $\left(w g^{1}\right)$ mutation on wing and haltere development in Drosophila melanogaster. Dev Biol 48: 461-465.

Siegfried E, Wilder EL, Perrimon N. 1994. Components of wingless signalling in Drosophila. Nature 367: 76-80.

Simcox AA, Roberts IJH, Hersperger H, Gribbin MC, Shearn A, Whittle JRS. 1989. Imaginal discs can be recovered from cultured embryos mutant for the segmentpolarity genes engrailed, naked and patched but not from wingless. Development 107: 715-722.

Simcox AA, Grumbling G, Schnepp B, Bennington-Mathias C, Hersperger E, Shearn A. 1996. Molecular, phenotypic, and expression analysis of vein, a gene required for growth of the Drosophila wing disc. Dev Biol 177: 475489.

St. Johnston D, Nuesslein-Volhard C. 1992. The origin of pattern and polarity in the Drosophila embryo. Cell 68: 201-219.

Strigini M, Cohen SM. 2000. Wingless gradient formation in the Drosophila wing. Curr Biol 10: 293-300.

Szuts D, Freeman M, Bienz M. 1997. Antagonism between EGFR and Wingless signalling in the larval cuticle of Drosophila. Development 124: 3209-3219.

Theisen H, Syed A, Nguyen BT, Lukacsovich T, Purcell J, Srivastava GP, Iron D, Gaudenz K, Nie Q, Wan FY, et al. 2007. Wingless directly represses DPP morphogen expression via an Armadillo/TCF/Brinker complex. PLoS ONE 2: e142.

Thuringer F, Bienz M. 1993. Indirect autoregulation of a homeotic Drosophila gene mediated by extracellular signaling. Proc Natl Acad Sci 90: 3899-3903.

van den Heuvel M, Nusse R, Johnston P, Lawrence PA. 1989. Distribution of the wingless gene product in Drosophila embryos: A protein involved in cell-cell communication. Cell 59: 739-749.

van de Wetering M, Cavallo R, Dooijes D, van Beest M, van Es J, Loureiro J, Ypma A, Hursh D, Jones T, Bejsovec A, et al. 1997. Armadillo coactivates transcription driven by the product of the Drosophila segment polarity gene dTCF. Cell 88: 789-799.

Waltzer L, Vandel L, Bienz M. 2001. Teashirt is required for transcriptional repression mediated by high Wingless levels. EMBO J 20: 137-145.

Wang SH, Simcox A, Campbell G. 2000. Dual role for Drosophila epidermal growth factor receptor signaling in early wing disc development. Genes Dev 14: 2271-2276.

Wiellette EL, Harding KW, Mace KA, Ronshaugen MR, Wang FY, McGinnis W. 1999. spen encodes an RNP motif 
protein that interacts with Hox pathways to repress the development of head-like sclerites in the Drosophila trunk. Development 126: 5373-5385.

Wieschaus E, Nüsslein-Volhard C, Jürgens G. 1984. Mutations affecting the pattern of larval cuticle in Drosophila melanogaster III: Zygotic loci on the X-chromosome and fourth chromosome. Rouxs Arch Dev Biol 193: 296-307.

Williams JA, Paddock SW, Carroll SB. 1993. Pattern formation in a secondary field: A hierarchy of regulatory genes subdivides the developing Drosophila wing disc into discrete subregions. Development 117: 571-584.

Williams JA, Paddock SW, Vorwerk K, Carroll SB. 1994. Organization of wing formation and induction of a wing-patterning gene at the dorsal/ventral compartment boundary. Nature 368: 299-305.

Wodarz A, Nusse R. 1998. Mechanisms of Wnt signaling in development. Annu Rev Cell Dev Biol 14: 59-88.

Zecca M, Struhl G. 2007a. Control of Drosophila wing growth by the vestigial quadrant enhancer. Development 134: $3011-3020$.

Zecca M, Struhl G. 2007b. Recruitment of cells into the Drosophila wing primordium by a feed-forward circuit of vestigial autoregulation. Development 134: 3001-3010.

Zecca M, Basler K, Struhl G. 1996. Direct and long-range action of a wingless morphogen gradient. Cell 87: 833844. 


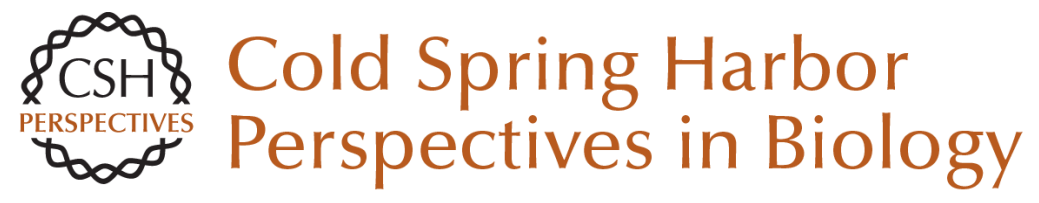

\section{Wnt/Wingless Signaling in Drosophila}

Sharan Swarup and Esther M. Verheyen

Cold Spring Harb Perspect Biol 2012; doi: 10.1101/cshperspect.a007930 originally published online April 25, 2012

\section{Subject Collection Wnt Signaling}

Wnt Signaling in Vertebrate Axis Specification Hiroki Hikasa and Sergei Y. Sokol

Secreted and Transmembrane Wnt Inhibitors and

Activators

Cristina-Maria Cruciat and Christof Niehrs

Wnt Signaling in Normal and Malignant

Hematopoiesis

William Lento, Kendra Congdon, Carlijn Voermans, et al.

Frizzled and LRP5/6 Receptors for Wnt/ $\beta$-Catenin

Signaling

Bryan T. MacDonald and Xi He

TCF/LEFs and Wnt Signaling in the Nucleus

Ken M. Cadigan and Marian L. Waterman

\section{Alternative Wnt Pathways and Receptors \\ Renée van Amerongen}

$\beta$-Catenin-Dependent Wnt Signaling in $C$. elegans:

Teaching an Old Dog a New Trick

Belinda M. Jackson and David M. Eisenmann

The Evolution of the Wnt Pathway

Thomas W. Holstein
The $\beta$-Catenin Destruction Complex Jennifer L. Stamos and William I. Weis

Wnt Signaling in Skin Development, Homeostasis, and Disease Xinhong Lim and Roel Nusse

Wnt Signaling in Bone Development and Disease:

Making Stronger Bone with Wnts Jean B. Regard, Zhendong Zhong, Bart O. Williams, et al.

Targeting Wnt Pathways in Disease Zachary F. Zimmerman, Randall T. Moon and Andy J. Chien

Wnt Signaling in Mammary Glands: Plastic Cell

Fates and Combinatorial Signaling Caroline M. Alexander, Shruti Goel, Saja A. Fakhraldeen, et al.

Wnt Signaling and Injury Repair Jemima L. Whyte, Andrew A. Smith and Jill A. Helms

Wnt Signaling and Forebrain Development Susan J. Harrison-Uy and Samuel J. Pleasure

Wnt Signaling in Neuromuscular Junction

Development

Kate Koles and Vivian Budnik

For additional articles in this collection, see http://cshperspectives.cshlp.org/cgi/collection/

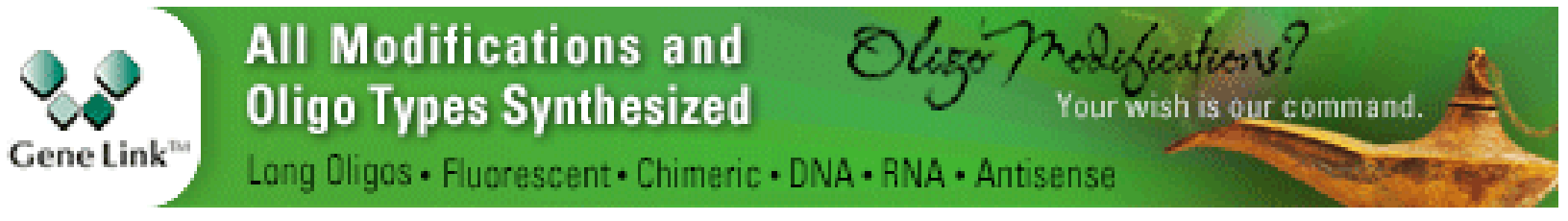

